\title{
Sparse Reconstruction Method for Separating Cardiac and Respiratory Components from Electrical Bioimpedance Measurements
}

\author{
Maksim Butsenko, Olev Martens, Andrei Krivosei, Yannick Le Moullec \\ Thomas Johann Seebeck Department of Electronics, Tallinn University of Technology, \\ Ehitajate tee 5, 19086, Tallinn, Estonia \\ maksim.butsenko@ttu.ee
}

\begin{abstract}
In this work, we investigate the possibility of employing sparse reconstruction framework for the separation of cardiac and respiratory signal components from the bioimpedance measurements. The signal decomposition is complicated by the nonstationarity of the signal and overlapping of their spectra. The signal has a harmonic structure, which is sparse in the spectral domain. We approach the problem by considering a dictionary with integrated wideband elements describing spectral components of the considered signal. The parameter estimation task is solved by the means of sparse reconstruction where solving the optimization problem returns a sparse vector of relevant dictionary atoms.
\end{abstract}

Index Terms-Electrical bioimpedance; Cardiac and respiratory components; Parameter estimation; Sparse reconstruction; Wideband dictionary.

\section{INTRODUCTION}

Electrical bioimpedance (EBI) measurements based applications for medical signal monitoring can provide interesting alternative to the conventional approaches due to noninvasiveness and cost-effectiveness. EBI measurements can characterize different properties of the human tissues and structures as well as various physiological dynamic processes in the human body, e.g. respiration and cardiac activities [1]. Medical applications can utilize EBI signal to analyze cardiac activities from simple heart-rate monitors [2] up to more sophisticated estimation of the cardiac output [3], [4] or central aortic blood pressure waveform [5]. EBIbased, noninvasive, continuous cardiac output monitoring can have several clinical applications in cardiology, emergency care, anesthesiology [3].

Extraction and separation of the cardiac and/or respiratory signal(s) is challenging; as these two signal components can vary significantly in time and frequency domains, they can have overlapping spectra and can be accompanied by severe noise, disturbances and artefacts. Respiratory and motionbased signal components can be considered as noise signals

Manuscript received 3 January, 2018; accepted 9 June, 2018.

This work was supported in part by the Estonian Research Council through the Institutional Research Project IUT19-11, by European Union through European Regional Development Fund and by the Horizon 2020 ERA-chair Grant Cognitive Electronics COEL H2020-WIDESPREAD2014-2 (Agreement number: 668995; project TTU code VFP15051). if one is only interested in the cardiac component. However, it is more interesting to try to extract both the cardiac and respiratory signals. Different approaches to this problem have been proposed in the recent literature including adaptive filtering [6], adaptive phase-locked loop [7], method based on the signal shape-locked-loop decomposer solution [8], principal and independent component analysis [9] and artificial neural networks [10]. These proposed methods are quite sophisticated; however, they either require considerable amount of fine-tuning and computational resources (neural networks) or require some time for converging to an optimal solution and exhibit low robustness to changes in the parameters of the signal (adaptive filters). Considering the variety of the proposed solutions, it is interesting to note that there is still no clearly established method available for extraction and separation of cardiac and respiration signal components, partly due to the problems mentioned above, but also partly due to the variations in the anatomy and physiology of human beings and their behaviour at various times and in physical and mental situations.

In this work, we are looking at employing a sparse signal processing technique for reconstructing respiratory and cardiac signals from EBI measurements (Fig. 1 shows an example of the EBI signal). The notion of sparsity usually implies that a signal is sparse in some domain (has only a few number of significant components). It has been noted that a large number of common applications results in signals that may be well approximated using a sparse reconstruction framework, and this area has seen increase in interest from the scientific community (see, e.g., [11], [12] and the references therein). A large part of these works has focused on convex algorithms that make use of different sparsity inducing penalties, which result in solutions that are well represented using only few elements from some (usually known) dictionary matrix, D. If the dictionary is appropriately chosen, it can be shown that even very limited measurements allow for an accurate signal reconstruction [13], [14]. We propose to reconstruct the relevant components of the EBI signal by considering wideband dictionary elements introduced in [15], [16]. These elements are formed as bands of frequencies covering the entire spectrum of interest. 


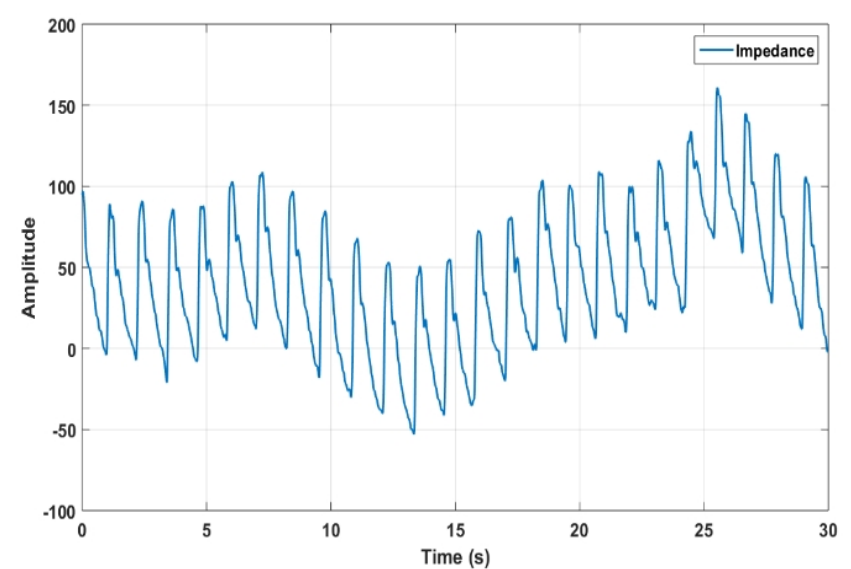

Fig. 1. Example of a waveform of an electrical bioimpedance measurements.

By refining the estimate through iteratively zooming into active parts of the spectra, the proposed algorithm is able to estimate signal parameters and reconstruct the signal without any prior knowledge.

\section{SpARSE RECONSTRUCTION}

The signal model for the considered EBI measurements can be written as

$$
y(t)=s_{C}(t)+s_{R}(t)+e(t)
$$

where $s_{C}(t)$ is the cardiac component, $s_{R}(t)$ respiratory component and $e(t)$ additive noise. We start with the assumption that both the cardiac and the respiratory components in the EBI signal are well approximated by a sum of sinusoids. Considering discrete-time signal consisting of $N$ samples, we can then reformulate (1) as

$$
y_{n}=\sum_{k=1}^{K} \sum_{l=1}^{L_{k}} \beta_{k, l} e^{2 i \pi f_{k} l t_{n}}+\varepsilon_{n}, \text { for } n=1, \ldots, N,
$$

where $K$ denotes the number of sources and $L_{k}$ the number of sinusoids for the $k$ th source. Furthermore, $f_{k}$ denotes the $k$ th fundamental frequency and $\beta_{k, l}$ the complex amplitude corresponding to the $l$ th harmonic of the $k$ th fundamental frequency, respectively, $t_{n}$ the $n$th sample time, and $\epsilon_{n}$ the additive noise at time $t_{n}$. In the problem at hand $K$ is known as we deal only with two sources. Hence, for reconstructing each source signal we can tackle the problem of estimating the frequencies $f_{k}$, for $k=1, \ldots, L_{k}$, of a measured signal $y_{n}$. The classical sparse formulation of this estimation problem, as presented in [17], considers the LASSO minimization [18]

$$
\min _{\mathbf{x}} \frac{1}{2}\|\mathbf{y}-\mathbf{D} \mathbf{x}\|_{2}^{2}+\lambda\|\mathbf{x}\|_{1},
$$

with:

$$
\begin{aligned}
& \mathbf{y}=\left[y_{1} \ldots y_{N}\right]^{T}, \\
& \mathbf{D}=\left[\begin{array}{ll}
\mathbf{d}_{1} \ldots \mathbf{d}_{P}
\end{array}\right], \\
& \mathbf{d}_{p}=\left[e^{2 i \pi \hat{f}_{p} t_{1}} \ldots e^{2 i \pi \hat{f}_{p} t_{N}}\right]^{T},
\end{aligned}
$$

where $\hat{f}_{p}$ for $p=1, \ldots, P$ denotes the $P \gg L_{k}$ candidate frequencies in the dictionary, $\mathbf{D}$, typically selected to be closely spaced to allow for minimal grid mismatch, and $(\cdot)^{T}$ the transpose. The penalty on the 1 -norm of $x$ will ensure that the found solution, $\hat{x}$, will be sparse, with $\lambda$ denoting a user parameter governing the desired sparsity level of the solution. The desired frequencies, as well as their order, are then found as the non-zero elements in $\hat{x}$. In this work we are limiting our approach to the use of LASSO minimization; however, in the literature there exist several well-known methods of solving undetermined system of linear equations (e.g. basis pursuit [19], orthogonal matching pursuit [20], etc.).

Deviating from the classical estimation method where the dictionary consists of closely spaced sinusoids, we propose exploiting integrated wideband dictionary elements in D [15], [16], such that each element is formed over the band

$$
\mathbf{d}_{b}\left(t_{n}\right)=\int_{f_{b}}^{f_{b+1}} e^{2 i \pi f t_{n}} d f=\frac{e^{2 i \pi f_{b+1} t_{1}}-e^{2 i \pi f_{b} t_{n}}}{2 i \pi t_{n}}
$$

as the resulting dictionary elements cover the band of frequencies from $f_{b}$ to $f_{b+1}$, for $b=1, \ldots, B_{l}$, the entire spectrum may be formed with $B_{1} \ll P$, where $B_{1}$ denotes the (initial) number of wideband dictionary elements. This approach coupled with iterative zooming into the active part of the spectra results in decreased computational complexity and improved estimation performance. For the in-depth discussion on the use and benefits of wideband dictionary elements based dictionaries we refer the reader to the comprehensive analysis in [16].

The resulting solution to the minimization in (3), $\hat{\mathbf{x}}$, can be further used to reconstruct an approximation of the initial EBI waveform as

$$
\hat{\mathbf{y}}=\mathbf{D}_{C} \hat{\mathbf{x}}_{C}+\mathbf{D}_{R} \hat{\mathbf{x}}_{R}
$$

where $\mathbf{D}_{C}$ and $\mathbf{D}_{R}$ denote dictionaries, while $\hat{\mathbf{x}}_{C}$ and $\hat{\mathbf{x}}_{R}$ denote solutions to the LASSO minimization for cardiac and respiratory components respectively.

\section{THE PROPOSED AlgORITHM}

We approach our analysis of the bioimpedance data by selecting two time windows $t_{c}$ and $t_{r}$, corresponding to the cardiac and the respiratory component respectively. Ideally we would like the time window to be close to the period of the analysed signal. A reasonable assumption for the rest rate of an adult is around 50-90 heartbeats per minute (bpm) for the cardiac component, which corresponds approximately to the heart rate with the frequency of $0.83 \ldots 1.5 \mathrm{~Hz}$; and around 10-18 breaths per minute for the respiratory component, which corresponds approximately to the respiratory rate with the frequency of $0.16 \ldots 0.30 \mathrm{~Hz}$. Correspondingly, we assign the time windows to be $t_{c}=1.5$ seconds and $t_{r}=6.25$ seconds. The respiratory component is reconstructed from the EBI data that is filtered through a 512-tap low-pass filter with cut-off frequency $f_{L P}=0.4 \mathrm{~Hz}$ 
and the cardiac component is reconstructed from the EBI data that is filtered through a 512-tap band-pass filter with $f_{B P 1}=0.7 \mathrm{~Hz}$ and $f_{B P 2}=3 \mathrm{~Hz}$. For each component we continue in a similar manner by analysing data for each time window. We solve the LASSO minimization in (3) using the initial dictionary $\mathbf{D}_{1}$ with $B_{1}$ number of elements constructed according to (7). Next, we discard the inactive bands of the spectra and solve the minimization task again now using the dictionary $\mathbf{D}_{2}$ with $B_{2}$ on parts of the spectra which were active in the first estimation step. By discarding inactive bands, we reduce the amount of computations required. In our experience, a two-step iterative zooming procedure (coupled with the proper choice of number of elements in the both dictionaries) is enough to achieve reasonable estimation precision; however, this choice is rather arbitrary. After the final estimation step, the algorithm returns a sparse vector which corresponds to the dictionary elements which fit the data in the best way. By extracting the fundamental frequency (first significant peak) from the vector, we get the corresponding rate of cardiac or respiratory part of the signal respectively. Then we move the window further on with an overlap of $50 \%$ with the previous data block. LASSO is solved through the alternating direction method of multipliers (ADMM) [21], derivation of the solution is presented in [15], [16], [22] and we refer the interested reader to description therein.

It has to be noted that using general LASSO formulation has a common weakness known as $f_{0}$ vs. $f_{0} / 2$ ambiguity [22] (here $f_{0}$ denotes the fundamental frequency). In some cases, the optimal solution to the minimization problem is going to be described similarly well (or even in a better way) by dictionary elements with fundamental frequency at half the actual frequency. As every other harmonic component in this solution will coincide with the actual signal component, then the algorithm returns $f_{0} / 2$ as fundamental frequency of the analysed signal. The problem is illustrated in the Fig. 2. The actual fundamental frequency of the cardiac signal as represented by ECG spectra is located at $0.83 \mathrm{~Hz}$. From the estimated signal spectra the first significant peak can be found at $0.44 \mathrm{~Hz}$ and second at $0.93 \mathrm{~Hz}$. It is clear that in this particular case the algorithm returned the fit with harmonic structure with fundamental frequency at $f_{0} / 2$ the actual one.

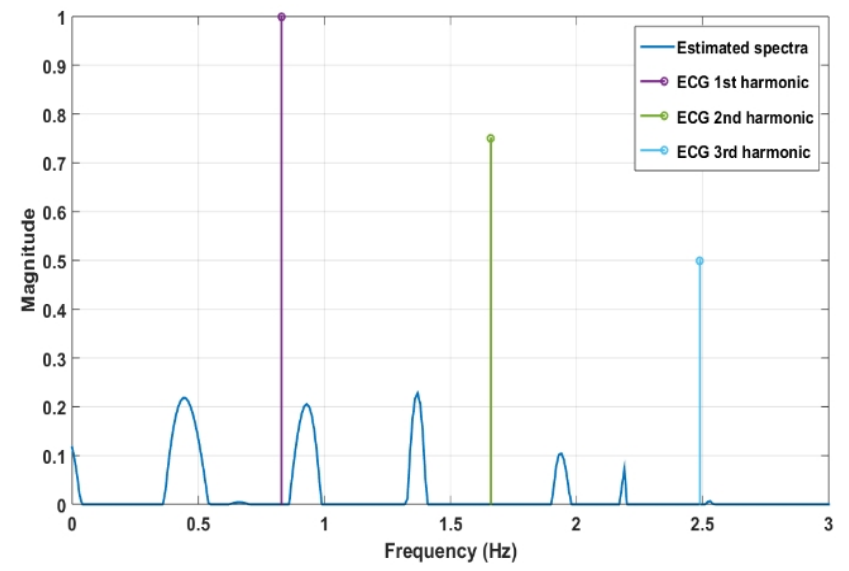

Fig. 2. Half fundamental frequency problem (magnitudes of ECG harmonics were chosen arbitrarily to show the location of the harmonics and do not reflect the actual values of the signal).
The currently proposed solution works reliably for the measurements where the cardiac component of the EBI signal lies in the frequency range of $0.8 \mathrm{~Hz}$ to $1.4 \mathrm{~Hz}$, corresponding to 48 to 84 heartbeats per minute. In this case $f_{0} / 2$ frequency lies well below the normal heart-rate of the human beings and can therefore be detected as wrong estimate and corrected accordingly. However, with the heartrate values above this range, $f_{0} / 2$ frequency will yield an estimate which falls in the range which can be considered to lie in the normal heartbeat range and therefore requires additional processing in terms of signal-tracking, which will exclude abrupt changes in the continuous estimation process. Proper remedy to this sort of situation can be found in the form of solutions which promote block-sparsity [22], [23], where the dictionary has an internal block structure and the final solution has to be block-sparse (i.e. use only few blocks to describe the solution).

\section{RECONSTRUCTION OF THE SIGNAL}

In our experiments for testing the proposed method, we used the collection of EBI signals recorded in clinical conditions. The EBI measurements were taken by using a CircMon device (JR Medical Ltd, Estonia) operating at a frequency of $30 \mathrm{kHz}$ and having a tetrapolar configuration of electrodes (together eight standard ECG electrodes on hands and feet). Initially the EBI signal is sampled at a rate of 1 $\mathrm{kHz}$ and is further decimated to $200 \mathrm{~Hz}$ using a 5-point averaging filter. All the simulations and analysis in this work were conducted in the MATLAB environment.

For the reconstruction, we have chosen to use a two-step estimation with $\mathrm{B}_{1}=100$ dictionary elements in the first estimation run and $\mathrm{B}_{2}=50$ dictionary elements in the second refining run. This choice results in the final resolution of $0.01 \mathrm{~Hz}$. The component reconstruction is carried out by multiplying the solution $\hat{\mathbf{x}}$ found by LASSO with the dictionary $\mathbf{D}$ for each time window $t_{c}$ or $t_{r}$. We can interpret the reconstructed block as a weighted sum of relevant dictionary elements. Figure 3 shows the separated respiratory and cardiac parts of the signal. As the reconstruction is done piecewise block by block, the breaks can be noted on reconstructed signal (especially on the respiratory component in Fig. 3). This can be mitigated by employing smoothing moving average filter for example. Fig. 4 shows the original EBI data and reconstructed EBI data which consists of reconstructed respiratory and cardiac components. In Fig. 5 we show the resulting fundamental frequency estimate of the cardiac signal component. We compare the result of the proposed method with the groundtruth, which is the frequency that is extracted from the ECG measurement done at the same time as EBI measurements. As we can see from the figure, the resulting estimate follows the actual frequency of the cardiac component closely and mean-square error (MSE) as compared to ECG measurements is 0.0007 .

Direct comparison with other algorithms is complicated by two factors. First, different authors use different metrics to evaluate efficacy of their approach. For example, classification metrics in [9], stroke volume estimation accuracy in [6] and in [10], visual comparison of original 
and reconstructed waveforms in [6] and [8]. Second and more important is the fact that different authors use different datasets (usually collected by their research group) and their measurements are not available for the wider research community, possibly due to privacy and ethical constraints related to the patient data. Authors tend to concentrate more on considering how their approach is able to capture the phenomena they are investigating. In our work the proposed algorithm is evaluated on 8 different EBI datasets measuring different levels of activity for different subjects.

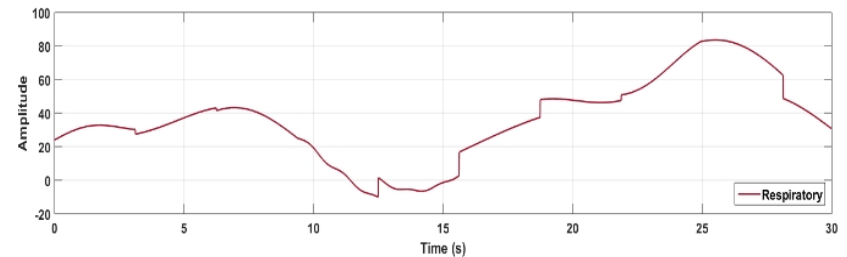

(a)

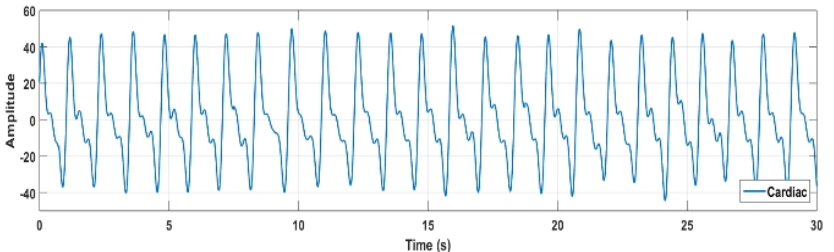

(b)

Fig. 3. Separated respiratory (a) and cardiac (b) signal components.

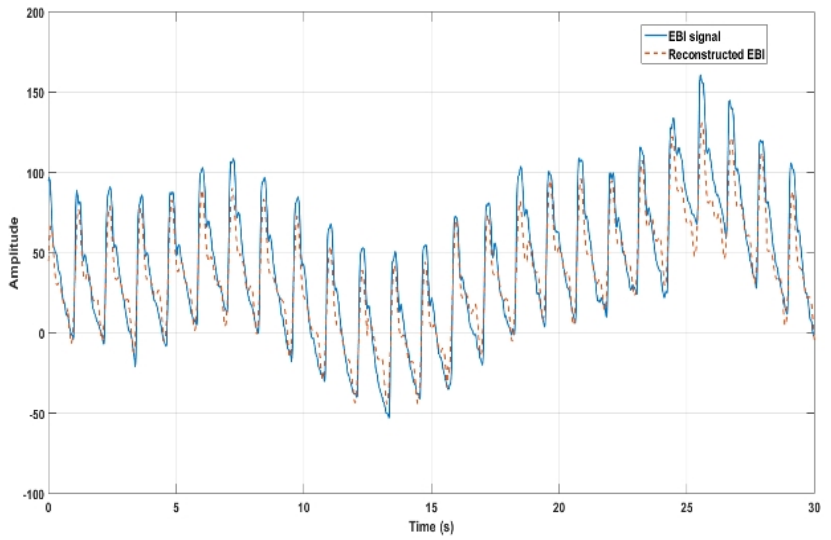

Fig. 4. Impedance signal as reconstructed from the sum of the separated cardiac and respiratory parts, compared to the original impedance signal.

For each set we have evaluated the proposed algorithm based on two MSE-s. Reconstruction MSE denoted as $\mathrm{MSE}_{R}$ (9) is calculated by comparing original EBI waveform with the waveform that is reconstructed from two separated signal components according to (8)

$$
M S E_{R}=\frac{1}{N} \sum_{n=1}^{N}\left(y_{n}-\hat{y}_{n}\right)^{2},
$$

where $y_{n}$ and $\hat{y}_{n}$ denote the $n$th sample of the EBI signal and reconstructed signal, respectively. Frequency estimation MSE denoted as $\mathrm{MSE}_{F}$ (10) is calculated by comparing frequency estimate of cardiac component with the ECG measurements which were collected simultaneously with the corresponding EBI dataset

$$
M S E_{F}=\frac{1}{M} \sum_{m=1}^{M}\left(f_{m}-\hat{f}_{m}\right)^{2},
$$

where $f_{m}$ denotes ground truth frequency and $\hat{f}_{m}$ frequency estimated by the proposed method. $M$ is the number of blocks for which the frequency estimate is calculated and depends on the length of the recorded signal, time window $t_{c}$ and window overlapping factor. Here we considered ECG measurements as the ground truth for the comparison. Results are presented in Table I.

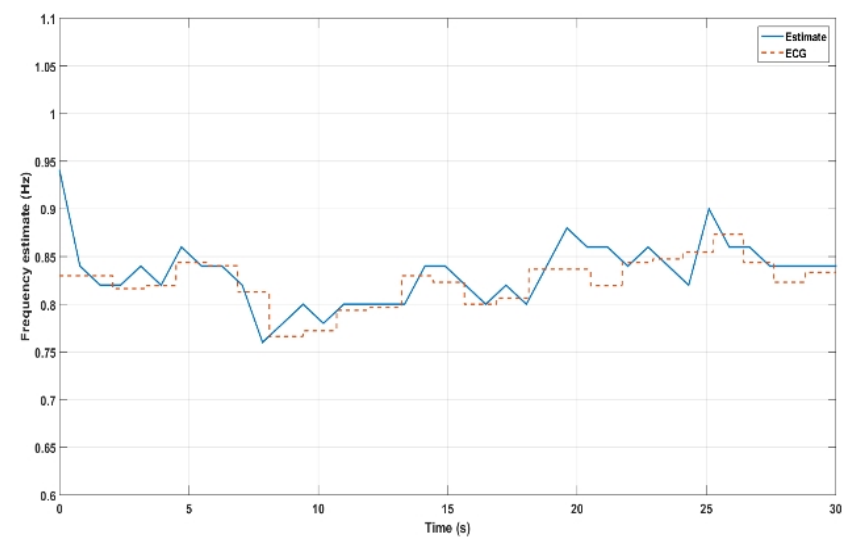

Fig. 5. Cardiac component frequency estimate compared to the frequency estimate as measured from ECG signal.

TABLE I. MEAN SQUARE ERRORS FOR RECONSTRUCTION OF EBI SIGNALS AND FREQUENCY ESTIMATE OF CORRESPONDING CARDIAC COMPONENT.

\begin{tabular}{|c|c|c|c|}
\hline EBI dataset name & Duration & MSE $_{\mathbf{R}}$ & MSE $_{\mathbf{F}}$ \\
\hline $02 \mathrm{ecg}$ & $107 \mathrm{~s}$ & $2.16 \mathrm{e}-06$ & 0.00129 \\
\hline $06 \mathrm{ecg}$ & $37 \mathrm{~s}$ & $5.09 \mathrm{e}-06$ & 0.00120 \\
\hline $07 \mathrm{ecg}$ & $67 \mathrm{~s}$ & $3.75 \mathrm{e}-06$ & 0.00658 \\
\hline $08 \mathrm{ecg}$ & $23 \mathrm{~s}$ & $2.97 \mathrm{e}-05$ & 0.00335 \\
\hline $12 \mathrm{ecg}$ & $117 \mathrm{~s}$ & $1.13 \mathrm{e}-06$ & 0.00251 \\
\hline $17 \mathrm{ecg}$ & $24 \mathrm{~s}$ & $3.88 \mathrm{e}-06$ & 0.00752 \\
\hline $101 \mathrm{ecg}$ & $103 \mathrm{~s}$ & $1.04 \mathrm{e}-05$ & 0.00540 \\
\hline $102 \mathrm{ecg}$ & $82 \mathrm{~s}$ & $3.90 \mathrm{e}-06$ & 0.00060 \\
\hline
\end{tabular}

\section{CONCLUSIONS}

In this work we have presented a novel method for separating cardiac and respiratory components from the electrical bioimpedance measurements. Signal components are recovered by the means of sparse reconstruction using the dictionary consisting of wideband elements. The proposed approach is able to reliably reconstruct both signal components and track fundamental frequency of cardiac component with high precision. Although the algorithm is not robust to the $f_{0}$ vs. $f_{0} / 2$ ambiguity problem, relevant postprocessing techniques provide a temporary remedy, while the planned extension to the proposed algorithm with the use of block-sparse dictionary structure should provide the necessary robustness in the future.

\section{ACKNOWLEDGMENT}

We would like to thank JR Medical Ltd (Tallinn, Estonia) and especially Jürgen Lamp for providing the EBI signal datasets that were used for our experiments.

\section{REFERENCES}

[1] S. Grimnes, O. Martinsen, Bioimpedance and Bioelectricity Basics. Academic Press, 2014.

[2] D. H. Diaz, O. Casas, R. Pallas-Areny, "Heart rate detection from 
single-foot plantar bioimpedance measurements in a weighing scale", in Annual Int. Conf. IEEE Engineering in Medicine and Biology Society (EMBC 2010), 2010, pp. 6489-6492.

[3] D. G. Jakovljevic, M. I. Trenell, G. A. MacGowan, "Bioimpedance and bioreactance methods for monitoring cardiac output", Best Practice \& Research Clinical Anaesthesiology, vol. 28, no. 4, pp. 381-394, 2014. DOI: 10.1016/j.bpa.2014.09.003

[4] B. Saugel, M. Cecconi, J. Y. Wagner, D. A. Reuter, "Noninvasive continuous cardiac output monitoring in perioperative and intensive care medicine", British Journal of Anaesthesia, vol. 114, no. 4 pp. 562-575, 2015. DOI: 10.1093/bja/aeu447.

[5] A. Krivoshei, H. Uuetoa, M. Min, P. Annus, T. Uuetoa, J. Lamp, "Cap waveform estimation from the measured electrical bioimpedance values: Patient's heart rate variability analysis", in 37th Annual Int. Conf. IEEE Engineering in Medicine and Biology Society (EMBC 2015), 2015, pp. 2788-2791. DOI: 10.1109/EMBC.2015.7318970.

[6] V. K. Pandey, P. C. Pandey, N. J. Burkule, L. R. Subramanyan, "Adaptive filtering for suppression of respiratory artifact in impedance cardiography”, in Annual Int. Conf. IEEE Engineering in Medicine and Biology Society (EMBC 2011), 2011, pp. 7932-7936. DOI: 10.1109/IEMBS.2011.6091956.

[7] A. Krivoshei, M. Min, T. Parve, A. Ronk, "An adaptive filtering system for separation of cardiac and respiratory components of bioimpedance signal", in IEEE International Workshop Medical Measurement and Applications (MeMea 2006), 2006, pp. 10-15. DOI: 10.1109/MEMEA.2006.1644448.

[8] A. Krivoshei, V. Kukk, M. Min, "Decomposition method of an electrical bio-impedance signal into cardiac and respiratory component", Physiological Measurement, vol. 29, no. 6, pp. 15-25, 2008. DOI: $10.1088 / 0967-3334 / 29 / 6 / \mathrm{S} 02$.

[9] I. Nejadgholi, M. Bolic, "A comparative study of pca, simca and cole model for classification of bioimpedance spectroscopy measurements", Elsevier Computers in Biology and Medicine, vol. 63, pp. 42-51, 2015. DOI: 10.1016/j.compbiomed.2015.05.004.

[10] S. M. M. Naidu, U. R. Bagal, P. C. Pandey, S. Hardas, N. D. Khambete, "Monitoring of stroke volume through impedance cardiography using an artificial neural network", in IEEE Twenty First National Conf. Communications (NCC 2015), 2015, pp. 1-6. DOI: 10.1109/NCC.2015.7084896.
[11] M. Unser, P. Tafti, An Introduction to Sparse Stochastic Processes Cambridge University Press, 2013.

[12] M. Elad, Sparse Redundant Representations. Springer, 2010. DOI: 10.1007/978-1-4419-7011-4.

[13] E. J. Candes, J. Romberg, T. Tao, "Robust uncertainty principles: exact signal reconstruction from highly incomplete frequency information", IEEE Trans. Inf. Theory, vol. 52, no. 2, pp. 489-509, 2006. DOI: 10.1109/TIT.2005.862083.

[14] D. L. Donoho, "Compressed sensing", IEEE Trans. Inf. Theory, vol. 52, pp. 1289-1306, 2006. DOI: 10.1109/TIT.2006.871582.

[15] M. Butsenko, J. Sward, A. Jakobsson, "Estimating sparse signals using integrated wide-band dictionaries", in 42nd IEEE Int. Conf. on Acoustics, Speech and Signal Processing, New Orleans, USA, 2017. DOI: 10.1109/ICASSP.2017.7952993.

[16] M. Butsenko, J. Swärd, A. Jakobsson, "Estimating Sparse Signals Using Integrated Wideband Dictionaries," Publication: eprint arXiv:1704.07584, 2017. DOI: 10.1109/TSP.2018.2835426.

[17] J. J. Fuchs, "On the use of sparse representations in the identification of line spectra", in 17th World Congress IFAC, Seoul, 2008, pp. 10225-10229. DOI: 10.3182/20080706-5-KR-1001.01730.

[18] R. Tibshirani, "Regression shrinkage and selection via the lasso", Journal of the Royal Statistical Society B, vol. 58, no. 1, pp. 267$288,1996$.

[19] S. Chen, D. Donoho, M. Saunders, "Atomic decomposition by basis pursuit”, SIAM review, vol. 43, no. 1, pp. 129-159, 2001. DOI $10.1137 / \mathrm{S} 003614450037906 \mathrm{X}$.

[20] J. Tropp, A. Gilbert, "Signal recovery from random measurements via orthogonal matching pursuit", IEEE Trans. Information Theory, vol. 53, no. 12, pp. 4655-4666, 2007. DOI: 10.1109/TIT.2007.909108.

[21] S. Boyd, N. Parikh, E. Chu, B. Peleato, J. Eckstein, "Distributed optimization and statistical learning via the alternating direction method of multipliers", Found. Trends Mach. Learn., vol. 3, no. 1, pp. 1-122, 2011. DOI: 10.1561/2200000016.

[22] S. I. Adalbjornsson, A. Jakobsson, M. G. Christensen, "Multi-pitch estimation exploiting block sparsity", Elsevier Signal Processing, vol. 109, pp. 236-247, 2015. DOI: 10.1016/j.sigpro.2014.10.014.

[23] N. Simon, J. Friedman, T. Hastie, R. Tibshirani, "A sparse-group lasso", Journal of Computational and Graphical Statistics, vol. 22 pp. 231-245, 2011. DOI: 10.1080/10618600.2012.681250. 